\title{
Performance of a diamond x-ray sensor fabricated with metal-less graphitic contacts
}

\author{
P. J. Sellin ${ }^{\text {a) }}$ and A. Galbiati \\ Department of Physics, University of Surrey, Guildford Surrey GU2 7XH, United Kingdom
}

(Received 30 March 2005; accepted 12 July 2005; published online 23 August 2005)

\begin{abstract}
We report the x-ray photocurrent response of a coplanar chemical vapor deposition diamond detector fabricated using a metal-less graphitic ohmic contact. Ion implantation of $70 \mathrm{keV}$ boron ions to a dose of $2 \times 10^{16} \mathrm{~cm}^{-2}$ was performed through a patterned photoresist to produce a coplanar graphitic contact structure. The device photocurrent showed a fast response to pulsed x-ray irradiation, and showed no evidence of photocurrent persistence that is observed in devices fabricated using metal Schottky contacts. The graphite-contact device also showed no extrinsic photoconductivity when illuminated with white light. () 2005 American Institute of Physics.

[DOI: $10.1063 / 1.2035885]$
\end{abstract}

Chemical vapor deposition (CVD) diamond has considerable potential for use as a radiation sensor, particularly for deep ultraviolet (UV) and x-ray detection. Various authors have reported investigations of coplanar CVD diamond detectors as a tissue equivalent radiation dosimeter, and have demonstrated an x-ray photocurrent sensitivity similar to that of silicon photodiodes. The unique properties of diamond are well suited for this application, including extreme radiation hardness, high resistivity, and good charge transport. However the development of practicable diamond $\mathrm{x}$-ray detectors has been restricted by a combination of priming effects and slow time response, or photocurrent persistence. These effects are due to a complex interplay between bulk trapping centers inherent in CVD material and the nature of the metaldiamond ohmic contacts used to fabricate the devices. In this work, we describe the performance of a coplanar x-ray sensor fabricated using a metal-less graphitized contact which was produced using a high dose implantation of boron ions. The interdigitated electrode structure was produced using only graphitized diamond, patterned by ion implantation through a photoresist.

Two coplanar diamond devices were fabricated, using free-standing $70 \mu \mathrm{m}$ thick undoped polycrystalline CVD diamond film grown by Element Six Ltd. Device 1 consisted of a single coplanar electrode structure, with an electrode width and interelectrode gap of $100 \mu \mathrm{m}$. The contacts were fabricated by a conventional photolithographic lift-off process, using thermally evaporated chromium $(50 \mathrm{~nm})$ followed by gold $(200 \mathrm{~nm})$. After lift off, the sample was annealed for $10 \mathrm{~min}$ at $400{ }^{\circ} \mathrm{C}$ in a nitrogen atmosphere to form a metal-carbide contact. Device 2 was fabricated using ion implantation with no metal contact. Prior to implantation, a $2.5 \mu \mathrm{m}$ thickness photoresist was spun onto the diamond and patterned using a conventional optical mask. The mask design was divided into four quadrants, containing four coplanar devices with a central common electrode and with electrode widths and interelectrode gaps of $5 \mu \mathrm{m}, 10 \mu \mathrm{m}$, $20 \mu \mathrm{m}$, and $50 \mu \mathrm{m}$ (Fig. 1). A broad beam ion implantation was applied to the device at room temperature using $70 \mathrm{keV}$ boron ions and a dose of $2 \times 10^{16} \mathrm{~cm}^{-2}$. After removal of the photoresist in acetone, the device was annealed for $4 \mathrm{~min}$ at

${ }^{a)}$ Electronic mail: p.sellin@surrey.ac.uk
$700{ }^{\circ} \mathrm{C}$ in a nitrogen atmosphere that resulted in a graphitic coplanar electrode structure. The measurements reported here for Device 2 were obtained from the quadrant with $20 \mu \mathrm{m}$ electrode spacing. After annealing, wire connections were made to the graphite electrodes using silver paint.

The x-ray photocurrent response of the two devices was characterized using a $30 \mathrm{kV}$ x-ray tube, operated at a maximum beam current of $500 \mu \mathrm{A}$. All measurements were taken at a bias voltage of $300 \mathrm{~V}$. Without $\mathrm{x}$-ray irradiation, the measured dark currents were $1 \mathrm{nA}$ and $0.2 \mathrm{nA}$ for Devices 1 and 2 , respectively. $\mathrm{X}$ rays were then applied using different beam currents in the range of $100-500 \mu \mathrm{A}$, with the beam pulsed on and off with a pulse duration of approximately 1 min. The corresponding dose rate received by the device was measured simultaneously using a calibrated ion chamber, and was in the range of $0.4-2.0 \mathrm{mGy} / \mathrm{s}$. Figure 2 shows the pulsed photocurrent data obtained from Device 1 with metallized contacts. The data show a linear increase in photocurrent as a function of dose rate, reaching a photocurrent of $0.07 \mu \mathrm{A}$ at $2 \mathrm{mGy} / \mathrm{s}$. Photocurrent persistence can be clearly observed in the data, with the current taking a finite time to reach a maximum value after each $\mathrm{x}$-ray pulse is switched on. A similar effect is observed each time the pulse is switched off, with the photocurrent decaying exponentially with a time constant of several minutes.

The data in Fig. 2 also show an increase in photocurrent when Device 1 is illuminated with white light. At a dose rate

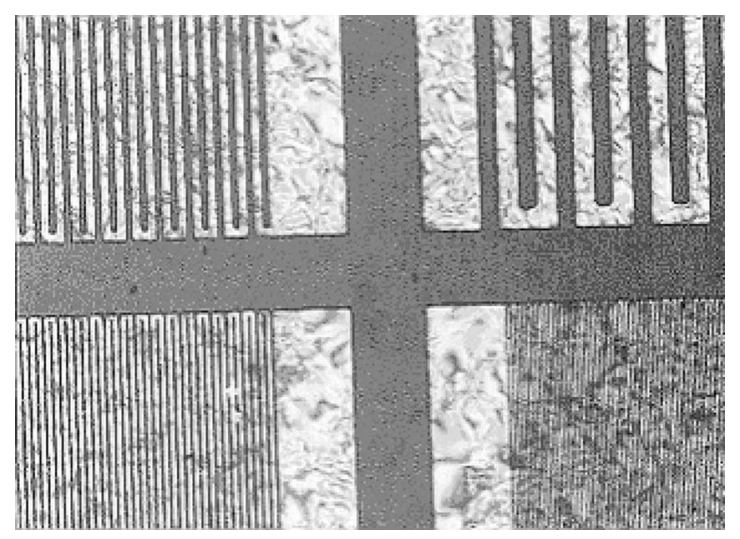

FIG. 1. Optical phase image contrast micrograph of the graphitized device, showing the four quadrants with electrode spacings of $5,10,20$, and $50 \mu \mathrm{m}$. 


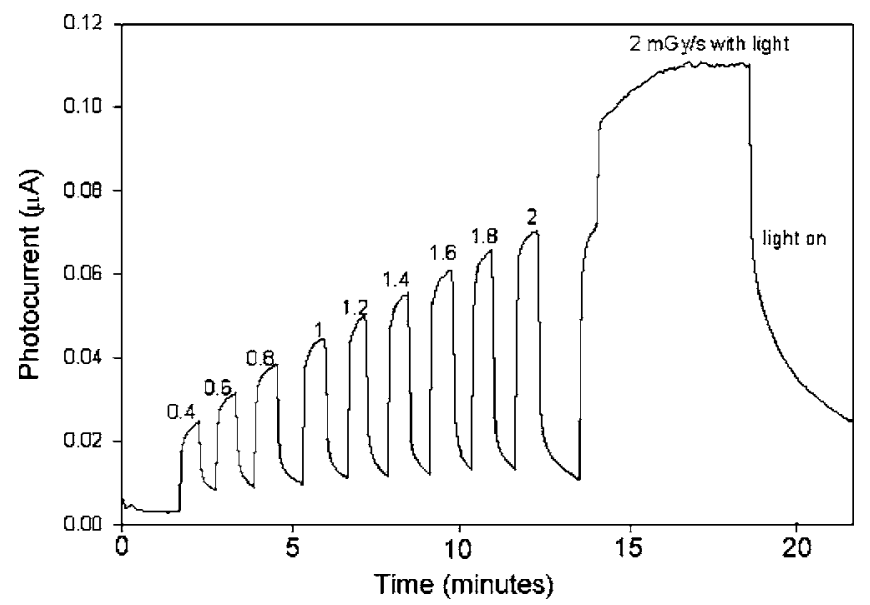

FIG. 2. Pulsed x-ray photocurrent from Device 1, fabricated using metallized contacts.

of $2 \mathrm{mGy} / \mathrm{s}$, the photocurrent increases from approximately $0.07 \mu \mathrm{A}$ with no illumination, to $0.11 \mu \mathrm{A}$ when illuminated. Extrinsic photoconductive sensitivity of coplanar CVD diamond devices has been reported by various authors, and is generally temperature dependent. Extrinsic photoconductivity may be due to various processes, including thermal ionization of states in the band gap or indirect phonon-assisted transitions to the conduction band. In our work, no attempt was made to suppress the extrinsic photocurrent sensitivity by using additional surface treatments.

Figure 3 shows the corresponding x-ray photocurrent data obtained from Device 2, fabricated using the metal-less graphitized contacts. The data show a linear increase in photocurrent as a function of dose rate, consistent with that obtained from Device 1, as shown in Fig. 4. The sensitivity of this device is $7.6 \mu \mathrm{C} / \mathrm{Gy}$, for photons with a peak energy of $30 \mathrm{keV}$, corresponding to a sensitivity per unit volume of $12 \mu \mathrm{C} /\left(\mathrm{Gy} \mathrm{mm}^{3}\right)$. Similar photocurrent performance and sensitivity data were also obtained for the graphitized device with $50 \mu \mathrm{m}$ electrode spacing.

A significant difference in performance between the metal contact and graphitized contact devices can be observed in terms of the photocurrent persistence. The graphitized device shows a prompt rise and fall of the photocurrent, following the time dependence of the x-ray pulses, and there is no evidence of any long time constant in the photocurrent

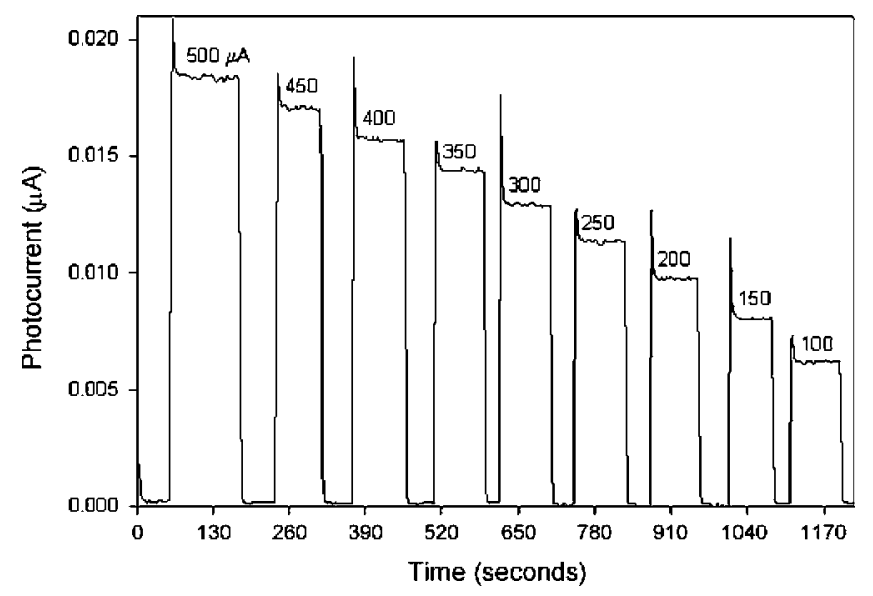

FIG. 3. Pulsed x-ray photocurrent from Device 2, fabricated using graphi-

tized contacts.
Downloaded 30 Mar 2009 to 131.227.178.132. Redistribution subject to AlP license or copyright; see http://apl.aip.org/ander illuminated and dark conditions.

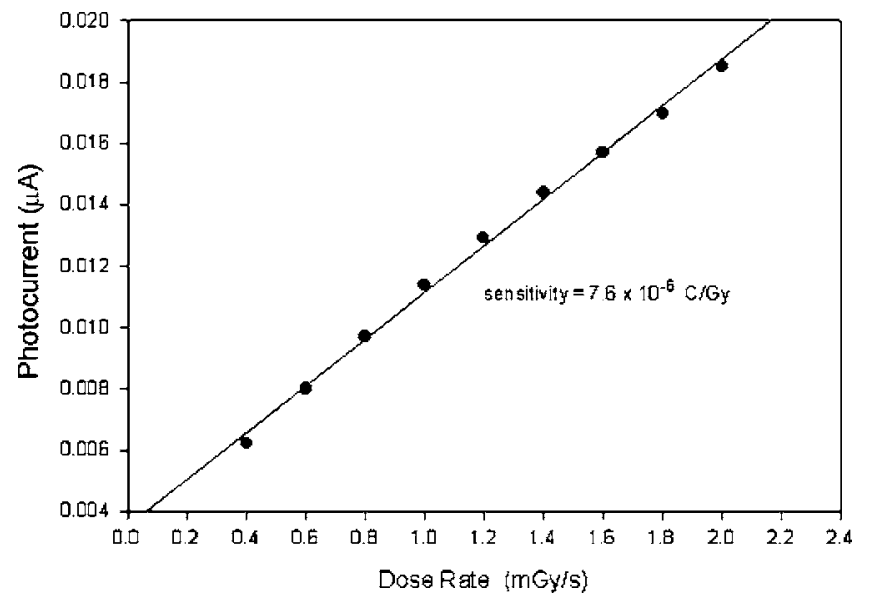

FIG. 4. X-ray sensitivity for Device 2, measured at $300 \mathrm{~V}$ bias.

behavior. A very prompt initial overshoot in the photocurrent is observed due to the resistance/capacitance time constant of the measurement circuit. When each X-ray pulse is switched off, the photocurrent returns immediately to the dark current value $(0.2 \mathrm{nA})$, and maintains this value after the irradiation sequence. Both devices were fabricated from CVD material of comparable quality, with a similar contact geometry differing only in electrode spacing. Consequently, the differences observed in the photocurrent persistence can be ascribed to the different methods of contact fabrication.

The x-ray photocurrents measured from the graphitized device show minimal sensitivity to white light, as indicated in Fig. 5. At an x-ray dose rate of $2 \mathrm{mGy} / \mathrm{s}$, a photocurrent of $18.3 \mathrm{nA}$ was observed, which was unaffected in terms of both amplitude and time response by illumination with white light. This is in marked contrast to Device 1, where whitelight illumination produced an $\sim 50 \%$ increase in $\mathrm{X}$-ray photocurrent (Fig. 2).

The x-ray photocurrent persistence that we observe in Device 1 is similar to that reported in natural type II-a diamond $^{1}$ in which decay time constants of $\sim 100 \mathrm{~s}$ were observed at room temperature due to detrapping from one or more traps that are active at room temperature. Previous authors have assigned such phenomena in undoped CVD diamond to the band of shallow hole traps centered at an energy approximately $0.61 \mathrm{eV}$ from the valance band. ${ }^{2,3}$ In contrast, photocurrent measurements from coplanar detectors using

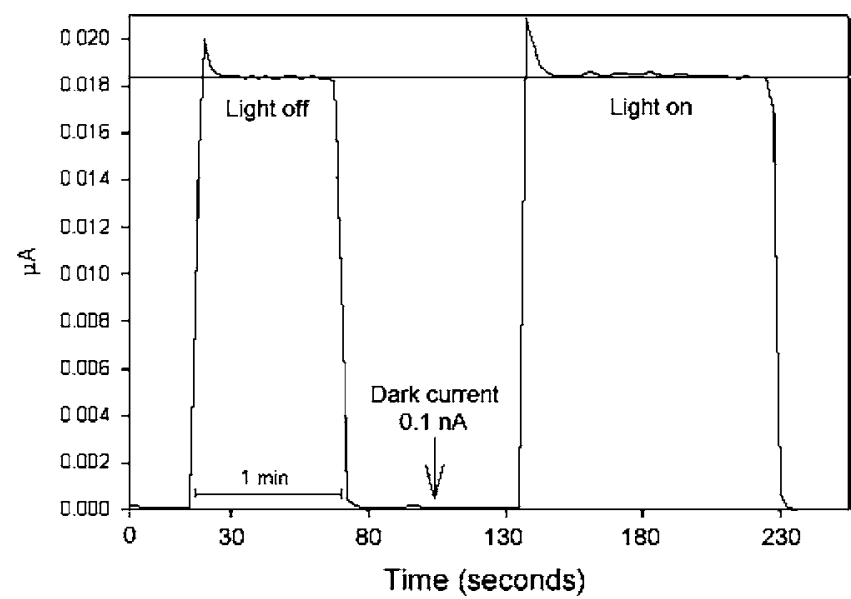

FIG. 5. Comparison of $\mathrm{x}$-ray photocurrent (500 $\mu \mathrm{A}$ beam current) obtained

from Device 2 under illuminated and dark conditions. 
fast pulsed UV lasers show a rapid time decay, consistent with carrier recombination lifetimes of $200-400$ ps. ${ }^{4}$ When illuminated with long pulses of UV light, coplanar diamond detectors also demonstrate significant photocurrent persistence. ${ }^{5}$ In one study using $200 \mathrm{~nm}$ irradiation from a deuterium lamp, a clear dependence was observed between photocurrent decay times and the nature of the metaldiamond contact structure. ${ }^{6}$ This indicated that the photocurrent decay time constant was strongly affected by the exact nature of the metal carbide/diamond contact system, in addition to the distribution of deep levels in the diamond bulk. The role of surface states in the generation of sub band-gap photocurrents has also been widely demonstrated in CVD diamond. Extrinsic photoconductivity has been suppressed by various treatments of the exposed diamond surface, for example, using methane ${ }^{7}$ or an oxygen plasma ${ }^{8}$ which remove $s p^{2}$ bonded carbon phases from the diamond surface.

These data demonstrate the viability of a metal-less ohmic contact on CVD diamond, based only on an ionimplanted graphitic layer. The ion implantation and annealing scheme used in this work used a dose of $2 \times 10^{16} \mathrm{~cm}^{-2}$ of $70 \mathrm{keV}$ boron ions, implanted at room temperature, followed by a $700{ }^{\circ} \mathrm{C}$ anneal for $4 \mathrm{~min}$. Graphitization of the diamond surface requires a critical implantation dose, which is dependent on both the ion type, energy, and implantation temperature. For room-temperature implantation of boron ions, this critical dose is typically $1 \times 10^{16} \mathrm{~cm}^{-2}$, below which the diamond can be restored to its original high resistivity state by annealing. At implantation temperatures below $320 \mathrm{~K}$, the mechanism for graphitization is, first, the amorphization of the diamond lattice, followed by the transition from $s p^{3}$ to $s p^{2}$ bonded carbon.

Venkatesan et al. ${ }^{9}$ demonstrated ion-implanted ohmic contacts fabricated on CVD diamond using a dose of 3.5 $\times 10^{16} \mathrm{~cm}^{-2}$. These structures contained a $0.2 \mu \mathrm{m}$ thick graphitized surface layer which was removed by etching and a Ti/Au evaporated layer added to produce a low resistance ohmic contact (with a specific contact resistance of 1 $\times 10^{-4} \Omega \mathrm{cm}^{-2}$ ). In our work, room-temperature ion implan- tation was used to minimize diffusion of radiation-induced interstitials. The annealing scheme was based on that reported by Zhang et al.; $;^{10}$ a 4 min anneal at $700{ }^{\circ} \mathrm{C}$ was chosen to ensure diffusion of boron ions from the interstitial diamond lattice sites and hence a low contact resistance. By contrast, annealing at too high a temperature, or for too long duration, can cause excess diffusion of boron to the surface.

Our results demonstrate that interdigitated detector structures can be successfully fabricated using a metal-less graphitized ohmic contact which combines ease of fabrication with improved performance due to the well-controlled doped interface between the graphitic surface and the diamond bulk. The use of a single-step photolithographic ion implantation, and the absence of metal lift off, considerably simplifies the fabrication of interdigitated diamond detectors. The graphitized devices did not show any photocurrent persistence when irradiated with $\mathrm{x}$ rays, which was in contrast to coplanar devices fabricated using metal-carbide contacts. Furthermore, the graphitized devices show almost no sensitivity to white light, consistent with a low concentration of shallow defects in the near-contact region.

${ }^{1}$ D. Tromson, P. Bergonzo, A. Brambilla, C. Mer, F. Foulon, and V. N. Amosov, J. Appl. Phys. 87, 3360 (2000).

${ }^{2}$ E. K. Souw, R. J. Meilunas, C. Szeles, N. M. Ravindra, and F. M. Tong, Diamond Relat. Mater. 6, 1157 (1997).

${ }^{3}$ J. Mort, M. A. Machonkin, and K. Okumura, Appl. Phys. Lett. 59, 455 (1991).

${ }^{4}$ F. Foulon, P. Bergonzo, C. Jany, A. Gicquel, and T. Pochet, Diamond Relat. Mater. 5, 732 (1996).

${ }^{5}$ A. Galbiati, Ph.D. thesis, University of Surrey, 2003.

${ }^{6}$ R. Di Benedetto, M. Marinelli, G. Messina, E. Milani, E. Pace, A. Paoletti, A. Pini, S. Santangelo, S. Scuderi, and A. Tucciarone, Diamond Relat. Mater. 10, 698 (2001).

${ }^{7}$ R. D. McKeag, R. D. Marshall, B. Baral, S. S. M. Chan, and R. B. Jackman, Diamond Relat. Mater. 6, 374 (1997).

${ }^{8}$ S. G. Wang, P. J. Sellin, and A. Lohstroh, Appl. Phys. Lett. 86, 093505 (2005).

${ }^{9}$ V. Venkatesan, D. M. Malta, K. Das, and A. M. Belu, J. Appl. Phys. 74, 1179 (1993).

${ }^{10}$ H. X. Zhang, Y. B. Jiang, Q. B. Meng, Y. J. Fei, P. R. Zhu, Z. Lin, and K. A. Feng, Appl. Surf. Sci. 150, 43 (1999). 